\title{
ArqueOlogía histórica en el Perú: POSIBILIDAdes y Perspectivas
}

\author{
Parker VanValkenburgh ${ }^{\mathrm{a}}$, Zachary Chase ${ }^{\mathrm{b}}$, Abel Traslaviña ${ }^{\mathrm{c}}$ y Brendan J. M. Weaver ${ }^{\mathrm{d}}$
}

\section{Introducción}

En el Perú actual, ¿qué es la «arqueología histórica»? La respuesta puede parecer algo simple: es el estudio, que emplea métodos arqueológicos, de entornos, momentos y procesos que también se encuentran registrados de manera contemporánea en textos escritos. Sin embargo, esta definición abarca una serie de preguntas adicionales, más implícitas: ¿Qué tipo de textos cuentan como suficientemente "históricos» y cómo están relacionados con la evidencia material? ¿Es que, en el acto de denominar a la arqueología histórica como un campo de estudio, estamos sugiriendo que debe haber algo diferente en la manera en que los arqueólogos estudiamos los restos, sitios y paisajes formados en las épocas colonial y republicana que en las épocas anteriores? Si es que sí, ¿a cuáles problemáticas se debe orientar?

En esta introducción, consideramos las implicaciones de estas preguntas para la arqueología histórica peruanista y los estudios andinos en general. Hasta la fecha, a pesar de que el patrimonio arqueológico peruano comprende numerosos sitios, monumentos y paisajes formados durante la Colonia y la época republicana, las investigaciones que han abordado estos lugares son relativamente escasas en comparación con el estudio de los restos del pasado precolombino. Sin embargo, en los últimos ańos, el interés académico en el patrimonio arqueológico colonial y republicano del Perú ha aumentado considerablemente. Aquí, en dos volúmenes del Boletín de Arqueología PUCP, presentamos veinte estudios nuevos que representan una muestra de la gama amplia de la arqueología histórica peruanista actual. Pretendemos mostrar que los estudios arqueológicos de sitios históricos ofrecen no solamente nuevas perspectivas y datos sobre la historia de los períodos colonial y republicano, sino también contribuciones íntegras a los estudios andinos en general.

El ímpetu para reunir esta colección de artículos se originó en el Simposio Internacional de Arqueología Histórica, un evento realizado en 2010 y organizado por los editores de estos dos volúmenes. Nuestra motivación para reunir el simposio se basó en dos realizaciones: i) que los arqueólogos peruanistas estaban haciendo aportes importantes al estudio de los períodos históricos, tanto en los Andes como el mundo Atlántico en general, y ii) que nuestros colegas, tanto en nuestra propia disciplina como en disciplinas corolarias, tenían poco conocimiento de nuestros esfuerzos. A propósito de levantar el perfil de estos trabajos y fomentar un diálogo interdisciplinario en torno a ellos, solicitamos doce ponencias y las emparejamos con una serie de comentarios de arqueólogos,

\footnotetext{
a Departamento de Antropología, Brown University

Correo electrónico: parker_vanvalkenburgh@brown.edu

b Brigham Young University

Correo electrónico: zachary_chase@byu.edu

c Departamento de Antropología, Vanderbilt University

Correo electrónico: abel.traslavina@vanderbilt.edu

d Departamento de Historia, Brea College

Correo electrónico: weaverb@berea.edu
} 
historiadores e historiadores de arte. Los trabajos fueron organizados en cuatro sesiones temáticas: transformaciones sociopolíticas durante la Colonia, estudios del espacio doméstico en el virreinato, producción y redes económicas, y la religión y evangelización durante la Colonia.

Mantenemos esa organización temática en esta publicación, pero hemos solicitado ocho trabajos adicionales a los originales para ampliar y diversificar la muestra de estudios aquí presentados. A pesar de su diversidad temática, teórica y metodológica, hay varios elementos que unen las visiones y agendas de estos artículos. Cada estudio emplea métodos arqueológicos e investiga la vida social en el mundo andino después de la invasión española. La gran mayoría consiste en estudios que están enfocados en sitios y paisajes situados dentro del territorio actual de la República del Perú, aunque también hemos incluido algunos trabajos dentro de los territorios actuales de Bolivia, Ecuador y Chile ${ }^{1}$. Esta decisión la hicimos a propósito por el hecho de que hay eventos y procesos que pasaron dentro del territorio del actual estado peruano, particularmente durante el período colonial, que no se pueden entender adecuadamente sin investigar sus conexiones con lugares y personajes cuyos trazos actualmente se encuentran dentro de los territorios de otros estados andinos ${ }^{2}$. Aparte de sus aspectos temporales y geográficos, esta colección también comparte enfoques metodológicos: cada autor analiza textos históricos junto a evidencias arqueológicas. No obstante, muchos de los estudios en esta colección también consideran evidencias de épocas anteriores, y otros también aprovechan las fuentes etnográficas y sociológicas.

En estos aspectos — la consideración de restos de varias épocas y fuentes de diversos orígenes-, estos trabajos no son nada fuera de lo común entre los estudios andinos. El transhistoricismo y la diversidad de las fuentes son aspectos íntegros de la arqueología peruanista, además de muchos trabajos etnohistóricos e historias del arte que trabajan en contextos andinos. Sin importar si nuestros objetos de estudio son cuevas del Período Arcaico, centros urbanos fechados en el Período Formativo o los restos de haciendas construidas en el siglo XVIII, nuestras interpretaciones del registro arqueológico están constantemente informadas por nuestra comprensión de las fuentes escritas. Desde los arqueólogos pioneros peruanos que trabajan en la tradición indigenista hasta los practicantes de las arqueologías autoconscientemente «científicas» $\mathrm{y}$ «sociales» del momento actual, todos empleamos de manera directa o indirecta los documentos escritos durante la época colonial y obras de etnografía para mejor entender los patrones que se manifiestan en el registro arqueológico prehistórico. En ese sentido, nos atrevemos a sugerir que todos los arqueólogos peruanistas practicamos alguna suerte de arqueología "histórica».

Hasta la fecha, sin embargo, pocos hemos considerado las implicaciones teóricas de nuestro empleo de las analogías históricas y etnográficas para interpretar el pasado prehispánico o los desafíos de acoplar la cultura material, textual y visual para narrar la historia. Un compromiso sostenido con la arqueología de las épocas históricas en el Perú requiere que nos hagamos estas preguntas y, también, hace posible buscar nuevas respuestas. Uno de los argumentos implícitos de esta colección es que la arqueología poscolombina ofrece no solamente nuevas «fuentes» y perspectivas para narrar la historia de la vida social en la Colonia y la época republicana, sino que también nos ofrece la oportunidad de generar una conversación crítica en torno a los métodos y teorías de los estudios históricos y arqueológicos andinos en general.

Si bien es cierto que el trabajo archivístico y el estudio de períodos históricos requiere otra formación académica y abarca temas distintos a la arqueología prehispánica, también es claro que hay un gran rango de preguntas y perspectivas que demandan que trascendamos las divisiones de los medios de comunicación y fechas calendáricas que definen la diferencia entre una época y otra. Por ejemplo, ¿cómo podemos entender el sistema económico de la Colonia y el transporte de bienes sin referencia a la construcción del Qhapac Nan, el sistema de caminos imperiales de los Wari, y a otros caminos y senderos «coloniales» de origen prehispánico? ¿Cómo damos sentido a la institución de la mita toledana sin apreciar las redes sociales y dependencias políticas creadas por la m’ita incaica? ¿Cómo estudiamos los cultos y oráculos coloniales sin apreciar sus precedentes precolombinos? En tales circunstancias, la división estricta entre campos de práctica arqueológica basada en la cronología y el carácter de las fuentes disponibles impide fundamentalmente la posibilidad 
de entender los objetos de nuestro estudio. No obstante, es cierto que la división institucional entre los estudios precolombinos y poscolombinos, reproducidos al nivel de muchas organizaciones profesionales y revistas académicas, sigue siendo una barrera sobresaliente que afecta a la práctica de la arqueología, particularmente en los Estados Unidos y Canadá (Funari 1999; Lightfoot 1995).

Por tales razones, sugerimos que la tendencia norteamericana de separar la arqueología "poscolonial» de la arqueología de otras épocas quizás no es deseable en el entorno peruanista. De la misma manera que la arqueología de las épocas precolombinas recurre a la interpretación de las fuentes históricas, la interpretación de los sitios y paisajes históricos (especialmente, durante la época colonial temprana) requiere que examinemos las raíces precolombinas de instituciones posteriores. A manera de comparación y orientación, presentamos en esta introducción una breve consideración de la definición y práctica de la arqueología histórica en el Perú. Luego, presentamos los primeros diez artículos en esta colección, y discutimos la manera en que su temática y sus enfoques teóricos y metodológicos sugieren varios futuros para la arqueología histórica peruanista.

\section{La arqueología histórica peruanista: precedentes y contextos}

Desde sus inicios, la arqueología peruanista ha sido informada por la lectura de textos históricos. En las obras pioneras Antigüedades peruanas (1851) de Mariano de Rivero y Johan Jacob von Tschudi, y El Perú (1874) de Antonio Raimondi, vemos que los autores recurrían a las crónicas coloniales como fuentes complementarias a los restos arqueológicos (Villacorta 2012; Tantaleán 2016). En su primera visita a Tiwanaku en 1890, Max Uhle y Alphons Stübel empleaban la crónica de Pedro Cieza de León como un punto de referencia cronológica para sugerir que el sitio fue ocupado antes del apogeo de los Inkas (Rowe 1998: 7).

Más adelante, algunos arqueólogos empleaban los textos de una forma más instrumental siguiendo sus huellas para localizar los restos de sitios o eventos mencionados en las crónicas. Según Juan Mogrovejo (1996) — cuyo trabajo sigue siendo el tomo historiográfico más comprensivo sobre las investigaciones de arqueología histórica en el Perú del siglo XX-, el primer intento moderno de realizar excavaciones sistemáticas en un sitio plenamente poscolonial fue realizado por José de la Riva Agüero y Osma en 1937, mediante sus esfuerzos por recuperar los restos de varias momias reales inka en el Hospital de San Andrés de Lima, documentados en la obra de Polo de Ondegardo, un proyecto que fue resucitado por Bauer y Coello (2007) en los años 2000, lamentable, sin que se encontraran los restos deseados.

A pesar del interés neto y diverso de la arqueología peruanista en los textos históricos, tomó tiempo para que emergiera una noción compartida de que los artefactos producidos o usados durante el período poscolonial pudieran en sí mismos valer la pena estudiar. La primera comunidad crítica del estudio de los restos de sitios históricos en el Perú no apareció hasta algunas décadas más tarde, en el Seminario de Arqueología de la Pontificia Universidad Católica del Perú, especialmente, el grupo liderado en la década de 1960 por Josefina Ramos de Cox. Durante estos primeros ańos, sus trabajos se concentraron en la interpretación sistemática de restos coloniales recuperados durante excavaciones en sitios cuyos elementos monumentales fueron erigidos durante la época prehispánica, como la Huaca Tres Palos y la Huaca Palomino (Cárdenas 1970, 1971, 1973; Cogorno 1970), y excavaciones en casas coloniales localizadas en el centro de Lima (Arrieta et al. 1975).

Es notable que estos avances fueron realizados en el Perú en el mismo momento en que la arqueología autoconscientemente «histórica» estaba emergiendo en otras regiones del mundo ${ }^{3}$. En el Reino Unido, la Society of Post-Medieval Archaeology (Sociedad de Arqueología Pos-Medieval) fue fundada en 1966 con la meta de fomentar el estudio arqueológico de la época medieval tardía hasta el período industrial en Gran Bretaña (Mytum 2016). Al año siguiente, la Society of Historical Archaeology (Sociedad de Arqueología Histórica) fue establecida por un grupo de estudiosos de los Estados Unidos, Canadá y México para seguir el mismo rumbo en América del Norte. Similarmente, la Australian Society for Historical Archaeology (ahora, Australasian) fue fundada en 1970. En varios aspectos, estos desarrollos podrían ser considerados parte del movimiento más 
amplio de preservación histórica, que en sí respondía a las amenazas percibidas por la «renovación urbana» posguerra al patrimonio histórico, particularmente, en los Estados Unidos.

Jamieson (2005) nota que, en paralelo, hubo un incremento en el interés de los estudiosos en otros países sudamericanos por los restos correspondientes al período colonial — por ejemplo, en Venezuela (v.g., Sanoja Obediente 1978) y Chile (v.g., Berdichewsky Scher y Calvo de Guzmán 1972) — D Durante las décadas de 1970 y 1980, sin embargo, la comunidad y práctica de la arqueología de los sitios históricos no se expandió en el Perú con la misma rapidez que se observó en otras regiones. Mientras que su crecimiento en los Estados Unidos, Canadá, Australia y el Reino Unido fue fomentado por cambios en las leyes de preservación histórica, no hubo cambios legales similares en el Perú que apoyaran financieramente el uso de la arqueología como método para preservar la cultura material colonial y republicana ${ }^{4}$.

En otros países sudamericanos, el perfil de la arqueología de sitios históricos creció notablemente durante la década de 1980. En artículos que revisan de varias maneras el estado e historia de la arqueología histórica sudamericana, Funari (1997, 2006), Pedrotta y Gómez Romero (1998), Politis (2003), Jamieson (2005) y Van Buren (2010) notan los aportes de colegas que trabajan en Colombia, Ecuador, Chile, Guyana y Venezuela; a la vez, subrayan el crecimiento notorio de la subdisciplina en Argentina, Brasil y Uruguay, tres países con historias poscoloniales altamente conectadas entre sí, cuyas identidades nacionales hacen referencias frecuentes a Europa (Funari 1997: 193, 2006: 202). Estos trabajos incluían las excavaciones extensivas llevadas a cabo por Daniel Schavelzon y otros en los contextos urbanos de Buenos Aires, Córdoba, Mendoza y Montevideo, entre otros sitios (v.g., Bárcena 1993; Fusco 1996 Schavelzon 1988, 1991, 1998, 2000) y la arqueología de misiones jesuíticas (v.g., Kern 1991, 1994; Poujade 1992; Zarankin 1995). Más recientemente, su enfoque ha sido ampliado para incorporar el estudio crítico de los quilombos (v.g. Guimarães 1992; Orser y Funari 2001; Funari 2003), y la arqueología de dictadura y resistencia en el siglo XX (v.g. Funari y Zarankin 2006).

Como indica Mogrovejo (1996), los trabajos en sitios históricos en el Perú continuaban durante este período, con sus mayores concentraciones en sitios encontrados dentro de contextos urbanos - particularmente, en iglesias y casonas localizadas en las ciudades de Lima, Cuzco y Trujillo, donde fueron llevados a cabo bajo proyectos de rescate. Adicionalmente, los arqueólogos que trabajaban en proyectos de investigación en mucho sitios prehispánicos seguían encontrando restos depositados durante las épocas colonial y republicana, reconocidos generalmente por elementos de notoria producción poscolonial, como la cerámica vidriada y las cuentas de vidrio (v.g., Beck et al. 1983; Donnan 2011). Lamentablemente, la mayor parte de la presentación de los resultados de estos trabajos se encuentra en la literatura "gris» —-mayormente, informes cortos depositados en los archivos del Instituto Nacional de Cultura, muchos de los cuales, según Mogrovejo, han desaparecido desde su presentación, y dejaron de fomentar una gran comunidad sostenida por el interés en el estudio de los restos arqueológicos poscoloniales-. La ausencia de una discusión sostenida en torno a estos materiales se contrasta con los estudios realizados contemporáneamente sobre la cerámica colonial por los historiadores del arte (Stastny 1981; Miasta Gutiérrez 1985, 2006; Acevedo 1986; Stastny y Acevedo 1986).

Una excepción notable es el proyecto Moquegua-Bodegas, dirigido por la doctora Prudence Rice entre 1985 y 1990, y dedicado al estudio de la vida social, la agricultura y el comercio de vino a través de excavaciones en varias de las bodegas abandonadas del valle de Moquegua fechadas entre los siglos XVII y XVIII. El proyecto resultó en la publicación de varios trabajos sobre estudios de la cerámica y la producción de vino (Rice 1994, 1996, 1997a, 1997b; Rice y Van Beck 1993; Smith 1997), y, más recientemente, trabajos expansivos de síntesis (Rice 2012a, 2012b, 2013). Adicionalmente, proveyeron un contexto para la formación de estudiantes doctorales estadounidenses, cuyos trabajos también se extendieron a la excavación de la reducción de Torata Alta en el valle de Moquegua (Smith 1991; DeFrance 1993; Van Buren 1993). A pesar de que su círculo de investigación consistía mayormente en extranjeros, el Proyecto Moquegua-Bodegas forjó redes sociales importantes para el desarrollo subsecuente de la arqueología histórica en el Perú y los Andes 
adyacentes, incluidas las excavaciones en Tarapaya y Porco (Bolivia), subsecuentemente dirigidas por Mary Van Buren.

Los artículos publicados en este volumen constituyen contribuciones nuevas de algunos de estos estudiosos pioneros, pero la mayor parte de los trabajos fueron escritos por autores que empezaron sus estudios de los materiales poscoloniales durante el siglo XXI, lo cual refleja el crecimiento dramático en la arqueología histórica del Perú en los últimos quince años, que destacan una nueva época en este campo de estudio. A pesar de este florecimiento, queda claro que la arqueología histórica todavía no está en el núcleo de la arqueología peruanista. La historia más actualizada de la arqueología peruana (Tantaleán 2016) no aborda el tema, mientras que un compendio amplio de artículos sobre la arqueología del continente sudamericano (Silverman e Isbell 2008) contiene solamente referencias pasajeras a trabajos de arqueología histórica. El deseo de buscarle un asiento en la mesa de los estudios andinos, en vez de considerarla como una iniciativa aparte, es una de las motivaciones principales para reunir esta colección.

\section{Temas y aportes: presentando y contextualizando la arqueología histórica en el Perú}

Como ya hemos aludido arriba, entre sus practicantes, no hay acuerdo preciso sobre las características que propiamente constituyen la arqueología histórica. Por supuesto, los criterios cronológicos, con sus procesos adjuntos ( $v$.g. la expansión de Europa y la propagación en marcha del capitalismo al nivel mundial), marcan todos los artículos de estos volúmenes. Sin embargo, estos también constan de la amplitud y variedad de lo que la arqueología histórica peruana brinda a los estudios académicos y otros intereses públicos. La división de los volúmenes por secciones temáticas refleja tanto las realidades sociohistóricas para los actores y grupos históricos como ciertos enfoques orientadores para arqueólogos actuales. Hay problemas teóricos centrales para la arqueología histórica mundial que son propios de cada sección, otros que corren entre artículos específicos y tras todo el contenido de los volúmenes. Más particularmente, en cuanto a la investigación del pasado andino, cada uno de los estudios representa intervenciones empíricas y teóricas. En lo que sigue, resumimos estas nuevas contribuciones de la arqueología histórica en cuatro categorías que corresponden al contenido y argumentos de los artículos: i) prácticas multi- o interdisciplinarias, ii) la detección de las actividades de grupos menos o no representados en el registro escrito, iii) nuevos marcos de interpretación y entendimiento, y iv) la relevancia del pasado en el mundo presente. Claro está el traslapo de todas las categorías; las separamos solo para facilitar la exposición de los principios.

Antes de elaborar esta discusión, es necesario primero revisar cuestiones que calan a las mismas bases del conocimiento del pasado, la investigación académica y otras empresas enredadas en la producción y reproducción del pasado. Desde un inicio, la arqueología de los sitios históricos provocaba discusiones críticas de la historicidad y la interpretación de los documentos históricos, pero no faltaban comentaristas que dudaban de la utilidad del oficio. Uno de los pioneros de la arqueología histórica norteamericana, James Deetz, una vez caracterizó en vena irónica la visión popular de la arqueología histórica como «la forma más costosa [...] de aprender algo que ya sabíamos» (1991: 1, traducción propia) ${ }^{5}$. Con esto, queremos subrayar la percepción de que si ya tenemos fuentes escritas que corresponden a un cierto período o fenómeno no vale la pena gastar recursos excavando los restos arqueológicos que corresponden al mismo período ${ }^{6}$. Tal suposición incurre una petición de principio de la epistemología histórica. Es decir, por tener acceso a escritos históricos, ¿qué fue lo que pensamos que ya sabíamos y qué justifica la convicción? ¿Cuáles son los detalles constitutivos de los procesos de la producción (y el «silenciamiento») de la historia y el pasado?

Como explica el antropólogo Michel-Rolph Trouillot (1995, 2003), la constitución de los hechos y narrativas históricas no es nada sencillo. Mejor dicho, abarca varios momentos críticos en que la voluntad de actores específicos ejerce una fuerte influencia en sí. Todo lo siguiente debe efectuarse: el reconocimiento de un evento o proceso como significativo; la creación de un registro de este; el acto de archivar los textos escritos; el reconocimiento, por parte de los estudiosos posteriores, 
de que los documentos de archivo contienen cuentos o información que es valiosa y relevante; la resonancia de los trabajos de estos estudiosos a través de la ratificación más amplia de sus interpretaciones y selecciones por parte de un público. El récord histórico, formado a través de procesos que pasan por cada uno de estos momentos, está compuesto no solamente por sus declaraciones afirmativas pero también por lo que no incluye, lo que Trouillot llama «silencios». Su exposición señala que el conocimiento histórico pasa por cadenas operativas y procesos tafonómicos, algo comparable con el registro arqueológico y su investigación. Esta comprensión permite que la arqueología histórica trate al pasado y sus rasgos con un ojo crítico y concienzudo.

$\mathrm{Al}$ mismo tiempo, los restos arqueológicos también son implicados en las complejidades que se suelen asociar con la producción e interpretación de los documentos escritos: limitaciones empíricas, sesgos políticos, económicos y sociales, la «inestabilidad del texto». Por ejemplo, Gabriel Ramón sugiere que la decoración superficial de alfares no necesariamente corresponde a las realidades sociopolíticas como frecuentemente hemos asumido que resuena con otros estudios recientes (ver también Makowski 2009; Druc 2013). Así, vemos que el registro arqueológico está formado a través de procesos similares a los expuestos por Trouillot y, por lo tanto, también abarcan silencios. Por ende, la arqueología histórica no puede llenar por completo lo que se ha perdido, olvidado o negado a «decir». No obstante, sí puede proporcionar líneas independientes de evidencia que echan luces más grandes sobre el pasado y también nos ofrecen una visión de realidades alternativas, como las de los individuos y poblaciones indígenas estudiados por Mary Van Buren, Parker VanValkenburgh, Abel Traslaviña, Steve Wernke y Haagen Klaus. Estas personas negociaban un mundo de costumbre colectiva y de nuevas expectativas coloniales; estos artículos exponen y analizan la variedad de formas en que se hizo. Otro ángulo complementario se ve en el estudio de Rosabella Álvarez-Calderón, en el cual demuestra que los tratamientos arqueológicos de las «ruinas», que llamamos «huacas» en Lima actual, han favorecido un ideal interpretativo de la modernidad —el propósito intencional de la construcción y el uso de complejos monumentales prehispánicos- y, por ende, no se ha prestado atención debido a la «historia negativa» de la «vida social» de las huacas durante la Colonia y la República. Es decir, la existencia de las huacas durante los últimos siglos ha sido «silenciada», aun cuando estas desempeñaron varios roles —entre ellos, la misma división chauvinista entre lo moderno y lo antiguo, lo prehistórico e histórico-. Álvarez-Calderón hace hincapié en estos roles para ampliar nuestro concepto de la urbanidad y la temporalidad.

Esto nos lleva a otra problemática para la cual la arqueología histórica oportunamente se relaciona con una división clásica de la historicidad. Muchos estudiosos ven a la distinción entre la prehistoria y la historia (y, por ende, la designación «arqueología histórica») como algo problemático (por ejemplo, Smail 2005, 2007 Christian 2011). En algunos círculos profesionales de historiadores, el término "prehistoria» ha llegado a ser una palabra casi tabú. Según este punto de vista, la historia es una cadena de eventos ininterrumpidos y la designación de la invención (o introducción) de la escritura como un rasgón en la tela del tiempo es, en el mejor de los casos, arbitrario y, en el peor, pernicioso, lo que refuerza el carácter monolítico del pasado antiguo, removiéndolo del espacio de la crítica (Thomas 2004; Smail 2007).

Estamos de acuerdo con este sentimiento y, en efecto, consideramos a la arqueología histórica como un puente que puede unir esta brecha, pero estamos menos preocupados sobre la terminología. Es claro que la incredulidad del historiador casi ofendido por el término "prehistoria» está parcialmente ligado a la ambigüedad que Trouillot consideraba inherente en la palabra "historia", el hecho de que el mismo término puede referir al flujo de eventos (el proceso sociohistórico) y la narración de esos eventos (lo que se sabe de lo que pasó). El historiador que exige que la arqueología se pliegue al proyecto histórico, demandando que el arqueólogo esencialmente tenga las mismas metas que el historiador (sino que solamente se dedica a interpretar otro tipo de evidencia) sugiere implícitamente que sublimemos nuestros objetivos a los suyos - que seamos sirvientes en la producción de la narrativa histórica, cuya voz, tempo y cadencia se derivan de la lectura de fuentes escritas- Consideramos que el aceptar estas condiciones nos impediría seguir varios de nuestros oficios más productivos: usar el estudio de la cultura material, la arquitectura, el espacio, y el paisaje 
para prever nuevas formas y estilos narrativos (Abercrombie 1998; Chase 2016, 2017; Dawdy 2016; Swenson y Roddick [eds.] 2017). De hecho, muchos de los fenómenos que los arqueólogos estudiamos — paisajes, sitios, instituciones y hasta personajes—, son transhistóricos. Es decir, su existencia y su eficacia no pueden ser estrictamente delimitadas y encajadas en un período. $\mathrm{Al}$ contrario, ejercen una influencia continua. Wernke lo ha encapsulado perfectamente cuando dio la denominación "transconquista» a su período de investigación, que comprende el preincaico tardío, el incanato y la Colonia española. Se ve también en el estímulo al estudio de largo plazo en los artículos de Rice, Van Buren, Fhon, Coello y Alvarez-Calderón; aparece en otra forma en la etnoarqueología de Ramón. Por otro lado, los elementos y procesos específicos de la construcción de los mundos pasado y presente provistos por los artículos de VanValkenburgh, Traslaviña, Ross Jamieson, Sofía Chacaltana, Brendan Weaver, Zach Chase, César W. Astuhuamán, Jeffrey Quilter y Melissa Murphy y María Fernanda Boza complican y dan matiz a los períodos, y al mismo concepto de periodizar que ha marcado nuestro conocimiento del pasado andino. La mayoría de estos estudios saldrán en el segundo volumen.

Cuestiones sobre qué tipos de fuentes cuentan históricamente y el papel social de la escritura (cf. Goody 1986, 2000; DeFrancis 1989) deben recorrer la arqueología histórica, particularmente en el Perú, dado que trabajamos en un contexto en que los medios de comunicación antes (y después) de la invasión española abarcaban tecnologías — más prominentemente, los khipuque parecen haber registrado y presentado al mundo de una forma distinta a los textos escritos (al respecto, véase Urton 2017). En efecto, la diversidad de la alfabetizaciones en los Andes, tanto durante la época prehispánica como durante la época colonial, ha inspirado a los estudiosos andinistas a producir una extensa y perspicaz literatura que investiga el carácter de la alfabetización, y la interacción de esta con otras formas de producir y representar lo sociohistórico (Salomon y Urioste 1991; Cummins 1994, 2002; Rappaport 1994; Rappaport y Cummins 1994, 2011; Arellano 1999; Hyland 2002, 2014; Quilter y Urton 2002; Salomon 2002, 2006; Brokaw 2010; Salomon y Niño-Murcia 2011; Urton y Chu 2015).

Estos temas aparecen en varias formas en los artículos contenidos en estos dos volúmenes. Además, resumimos las contribuciones de los estudios en cuatro categorías:

i) Como se debe imaginar, una de las ventajas de la arqueología histórica es su insistencia en el uso crítico de los datos provenientes de las fuentes tradicionales de varias disciplinas académicas y, como mencionamos, los estudios andinos han compartido esta misma tendencia. No obstante, en el pasado, esta tendencia ha resultado en una insularidad cultural por la cual, como escribe Mary Van Buren, «una oportunidad de crear una verdadera antropología histórica interdisciplinaria se dejó pasar» (este número). La aspiración de Van Buren es el desarrollo de una empresa académica no solo fiel al pasado, sino también relevante al presente (tratado con más detalle posteriormente). Su visión interdisciplinaria suena con los enfoques sugeridos por la mayoría de los demás autores de estos volúmenes ${ }^{7}$. Prudence Rice aboga por «el nuevo campo de la ecología política» para iluminar la producción y el mercado transatlánticos de loza vidriada. Los análisis de espacio y de arquitectura, característicos de investigaciones de paisaje y ampliados por los Sistemas de Información Geográfica (SIG), son las bases de los estudios de Steve Wernke y Abel Traslaviña sobre asentamientos indígenas en la sierra arequipeña durante la Colonia temprana. A través de la investigación etnográfica de la producción de alfarería actual, Gabriel Ramón propone modelos para mejor entender las ollas y personas del pasado. Por su parte, la pericia de Haagen Klaus en bioarqueología le permite proveer datos de cantidad y precisión impresionantes sobre el descenso de la salud entre poblaciones indígenas durante la Colonia. Una multitud de otros ejemplos también se encuentran en los artículos del segundo volumen. Lo que Van Buren y los demás autores de la sección teórica y la sección de "poder, dominación y resistencia» ofrecen son nuevas formas de continuar, hasta ampliar, la ruta multidisciplinaria, y, a la vez, evitar esencialismo cultural o ahistoricismo.

Por supuesto, combinar los registros escrito y arqueológico es la interdisciplinaridad más básica y penetrante de la arqueología histórica. Cabe mencionar que el hallar nuevos datos escritos no es exclusivo de las investigaciones archivísticas (aunque, por supuesto, queda bastante por encontrar 
y examinar en los archivos): proyectos de arqueología histórica han resultado hasta en descubrimientos de nuevos documentos (v.g. Cogorno Ventura 1970). En un caso recientemente publicado (Quilter et al. 2010) por un equipo que incluye a dos de los participantes del SIAH (Jeffrey Quilter y Karen Spalding), el hallazgo de un documento resultó en el redescubrimiento de lo que parece ser un idioma andino previamente perdido o desconocido por escritura. Aunque el argumento de Van Buren apela a un cambio de vista muy amplio y sofisticado, los casos mencionados nos hacen recordar la interdependencia de la arqueología y la historia en una forma más empíricamente básica. El punto no debe ser disminuido. El aumento de datos empíricos en ambos registros, arqueológico y escrito, será el mero avance de la arqueología histórica en el Perú. Recordemos la caracterización ingenua y simplista de la arqueología histórica dada por James Deetz (que investigaciones arqueológicas son un malgaste de recursos si tenemos acceso a escritos históricos provenientes del lugar y período en cuestión). Consideramos que las investigaciones arqueológicas de sitios históricos han desmentido esta concepción desde hace mucho tiempo. Proponemos, además, que es posible que sea precisamente este prejuicio — que los estudios arqueológicos son innecesarios o frívolos en tales contextos, y requieren una justificación explícita - el que ha fomentado un grado excepcional de introspección crítica y claridad de propósito entre los arqueólogos que tratan temas históricos.

Hasta en los casos en que los datos históricos y arqueológicos parecen ser mutuamente concordantes, la arqueología puede proporcionar detalles (e incluso datos) que nos facilitan tocar nuevas preguntas fundamentales, tales como las formas diferentes en que los procesos sociales se llevaron a cabo y sus dimensiones afectivas. En los casos que los registros arqueológicos e históricos no coinciden, su discordia abre la posibilidad de investigar críticamente no solamente los procesos históricos, sino también el proceso en que los narramos.

Sin duda, avanzar más allá de descubrir nueva información no es nada sencillo, pues implica la consideración de la gama de cuestiones de la semiótica, la cultura y los contextos históricos particulares. Sin embargo, también, promete revelaciones valiosas — descubrimientos que puedan cambiar nuestro entendimiento del pasado andino y de nuestro propio mundo- . Pensemos de nuevo en los khipu. Aparte de unir entre sí a la cultura material y la información histórica, recientes y asombrosos avances hacia posibles «lecturas» de estos registros andinos han sido posibles gracias a la combinación de investigaciones arqueológicas, históricas y etnográficas (Salomon 2006; Urton y Brezine 2005; Urton 2008; 2017; Salomon y Niño-Murcia 2011; Curatola Petrocchi y de la Puente Luna 2013; Giersz y Pardo [eds.] 2014; Urton y Chu 2015). En una vena similar, José Luis Pino (2014; véase también Lecoq 2013) reúne el análisis arqueológico en Huánuco Pampa con el análisis de registros históricos escritos y gráficos (como los escritos y dibujos de Guamán Poma o de Martín de Murúa) para proponer una interpretación sobre la semiótica y cosmovisión de los incas. En breve, el énfasis de la arqueología histórica en la utilización crítica de una variedad de fuentes de datos es propicia para el estudio del pasado andino, porque facilita el redescubrimiento de realidades cuyas huellas cayeron entre varios registros durante los últimos seis siglos. No obstante, no se debe olvidar que durante el mismo lapso también hubo personas — sectores enteros de la población andina- que cayeron por las grietas de la historia.

ii) Una faceta muy mencionada por sus practicantes es que la arqueología histórica ofrece la posibilidad de detectar las actividades de grupos menos o no representados en el registro escrito. De forma general, se ve en las «otras historias» $\mathrm{u}$ «otras narrativas» que se han mencionado previamente; ejemplos más específicos son los posibles «moriscos alfareros», «espías indígenas» de México y los bienes de contrabando mencionados por Rice. Lo que sabemos por contornos históricos amplios en cuanto al impacto del arribo de los espańoles en la vida, salud y muerte de la gente indígena del Perú se expone de manera concreta por el estudio bioarqueológico de restos óseos de Klaus, y Murphy y Boza. En otro caso, a pesar de tener relativamente poca información escrita sobre el tema, el análisis de Wernke demuestra cómo el medio ambiente construido formó y reflejó los cambios que acompañaron la llegada de los franciscanos en un pequeño asentamiento en el Colca. Por su parte, Van Buren extiende sus ya bien recibidas investigaciones (1996) sobre los cambios $\mathrm{y}$ continuidades en formas culturales en vista de diferentes contextos históricos y las decisiones 
estratégicas de individuos; aquí demuestra que, pese a los cambios en la minería boliviana sobre el largo plazo, han sido caracterizados por una pequeña escala y «el k'aqcheco —el robo de mineral de alta calidad». Estas nuevas perspectivas surgieron gracias a la investigación arqueológica.

iii) Las ventajas tratadas en estas primeras dos categorías demuestran cómo la arqueología histórica lleva a la formulación de nuevos marcos de interpretación y entendimiento del pasado peruano. Como vemos, la arqueología histórica ha tendido a ser un frente delantero en el desarrollo de nuevos marcos teóricos para la mejor reconstrucción y entendimiento del pasado y del presente. De esta manera, la subdisciplina se esmera en empujar la interdisciplinaridad de estudios andinos hacia las metas de interpretación y reflexividad que se ven en otros enfoques críticos, como estudios de la cultura material, del paisaje, o de la temporalidad y la historicidad. Por ejemplo, durante el SIAH, Chase introdujo la utilidad del concepto semiótico de performativity ${ }^{8}$, para tocar los temas de la evangelización y conversión. Como respuesta, el historiador Juan Carlos Estenssoro estimó que el concepto era indispensable para entender el pasado colonial del Perú, debido a que la entera empresa colonial era construida a partir de intentos de controlar el conocimiento y la percepción de la realidad (el presente y el pasado) a través de aserciones hechas en el espacio/paisaje, la cultura material, el lenguaje, las tradiciones y prácticas colectivas (véase también Chase 2015, 2017). Por lo tanto, como escribe VanValkenburgh, "[e]n lugar de reflejar, pasivamente, las divisiones [...] socio-políticas, las fuentes históricas y la arqueología participan directamente en su construcción». Para Chase y VanValkenburgh, el enfoque también se aplica a las fuentes escritas y el registro arqueológico. Sin este entendimiento, es probable que nuestras investigaciones simplemente sostengan las contenciones de los poderosos del pasado.

iv) Este reconocimiento de la construcción del pasado y el presente está relacionado con un interés que la arqueología histórica comparte con el resto de la «arqueología crítica», a saber, la relevancia y utilidad del conocimiento del pasado para la vida actual. Este interés se expresa en varias formas: que la arqueología sea algo más que una búsqueda de información para satisfacer la curiosidad desinteresada, que tenga impacto en la política del presente, que sea de beneficio material. Consideremos algunas de estas expresiones.

Anticipamos que la arqueología de sitios históricos continúe haciendo contribuciones vitales al estudio del mundo atlántico, más la arqueología histórica andina también está en una posición de poder hacer intervenciones únicas en las conversaciones de procesos culturales andinos. Creemos que, por cultivar estas conversaciones con académicos de los campos de la historia, la antropología, historia del arte y otros arqueólogos que investigan períodos más tempranos de la experiencia andina, la arqueología histórica andina tendrá contribuciones críticas al entendimiento de la experiencia social en el pasado.

Desde la década de 1990, la subdisciplina se ha enfocado más y más en el estudio crítico de los «raíces del presente» - el proyecto de examinar críticamente a la formación de las configuraciones hegemónicas de género, clase, raza, etnicidad, y otros asuntos sociopolíticos y económicos del mundo contemporáneo- (v.g. Ferguson 1992, Leone et al. 1987, McGuire 1992, Scott 1994, Singleton 1995). De hecho, el estado de misión oficial de la SHA define su campo de práctica como «la emergencia, transformación y carácter del mundo Moderno,» tomando el año 1400 d.C. como un punto de partida oficial. Por otro lado, hay practicantes que han sugerido que la mejor manera en que la arqueología puede erosionar las estructuras hegemónicas del poder es de colaborar con el público fuera de la arqueología para identificar tópicos de interés urgente y orientar nuestros esfuerzos a investigar los restos de la vida contemporánea que están relacionados con ellos (v.g. Little y Shackel 2007, McGuire 2008, Zimmerman et al. 2010; Atalay et al. 2014; De León 2015).

La arqueología histórica andina puede contribuir a fortalecer el sentimiento del patrimonio nacional. Existe una identificación profunda con los períodos de la Colonia y de la República temprana, expresada con fuerza especial en los centros urbanos del Perú. A través del aumento del estudio de las concretas bases históricas de los sentimientos de patrimonio de los peruanos, de darles matiz, hasta también desafiar algunas de las nociones y los sentimientos populares, la arqueología histórica puede atraer la atención del público y animarlo a participar en la reproducción democrática del patrimonio. 
Finalmente, la arqueología histórica puede hacer contribuciones materiales a través de aumentar el turismo, tanto nacional como internacional. Las investigaciones de la arqueología histórica como las expuestas por los artículos de Fhon y Coello tienen la promesa de incitar el interés de los peruanos, y se encuentran dentro de los centros urbanos mencionados arriba, lo cual significa que son accesibles para visitas casuales y repetidas, diferente a muchos de los sitios arqueológicos prehispánicos. Además, estos sitios son menos difíciles de proteger del huaqueo.

Claro está el hecho de que los magníficos sitios prehistóricos del Perú y los otros países andinos mantendrán su estatus como lugares de mayor atracción turística internacional y nacional, y debidamente. Al mismo tiempo, se puede ver en las catacumbas franciscanas de Lima; el Museo de la Inquisición que mantiene el Congreso Peruano; la Fortaleza del Real Felipe en Callao; o la sección histórica del Museo Nacional de Antropología, Arqueología e Historia de Pueblo Libre lo que también se nota en México, Europa, los Estados Unidos, Canadá, Japón y varias otras partes del mundo: sitios arqueológicos y hasta parques enteros basados en períodos históricos son algunos de los sitios arqueológicos más populares (y, en algunos casos, más visitados), particularmente por escolares y otros «turistas ciudadanos» que, por la proximidad y facilidad de acceso, pueden realizar repetidas visitas, como hemos indicado previamente.

Si bien los artículos en estos dos volúmenes reflejan una gama amplia de intereses y orientaciones para la arqueología histórica peruanista, todavía quedan muchos temas por investigar, en particular, la arqueología de la presencia africana y asiática en el Perú y sus conexiones diaspóricas. En el caso de la diáspora africana, los estudios históricos realizados desde la década 1970 han arrojado mucha luz sobre las poblaciones esclavizadas y libres, y los individuos de ascendencia africana, tanto dentro de las fronteras del virreinato colonial como en la joven república peruana (v.g. Millones Santagadea 1971, 1973; Harth-Terré 1973; Cushner 1975; Crespo 1977; Bowser 1974; Lane 2002; Aguirre 2005; Brockington 2006; Jouve Martín 2008; Arrelucea Barrantes 2009; McKinley 2010, 2012, 2014; O’Toole 2012). Sin embargo, la diáspora africana y las comunidades asiáticas en el Perú siguen siendo insuficientemente representadas en la literatura histórica (Andrews 2016). La arqueología de los sitios históricos está idealmente posicionada para abordar esta deficiencia.

No dudamos que el interés arqueológico reciente en las importantes oleadas de inmigración desde China al Perú en el siglo XIX resultará fructífero para una mejor comprensión de la economía política del Mundo Pacífico y el intercambio cultural en las primeras décadas del Perú republicano (véase Chuhue et al. 2012). Por otro lado, el lanzamiento en 2012 del Proyecto Arqueológico Haciendas de Nasca (PAHN) representa un esfuerzo sostenido de comprender la etnogénesis afroperuana y la esclavitud agroindustrial en el Perú a través del estudio de la cultura material de las poblaciones afrodescendientes esclavizadas en los viñedos jesuitas de los siglos XVII y XVIII en Nasca (Weaver 2015, 2016). Esperamos que pronto haya otros proyectos arqueológicos enfocados en el estudio de la diáspora afroandina en el Perú, puesto que la arqueología histórica ha hecho aportes críticos a su estudio en otras partes de las Américas y el mundo atlántico (Singleton y Torres de Souza 2010; Funari y Orser [eds.] 2015).

\section{Conclusión}

Hay cierto encanto que acompaña la experiencia de ver y tocar materiales que son mencionados en documentos, libros y otros textos históricos. En formas más sofisticadas, la arqueología histórica ofrece al mundo científico/académico nuevas vías de conceptualizar el pasado que investiga, fenómeno expresado durante el Simposio en 2010 por comentaristas historiadores, etnógrafos, historiadores de arte en sus reacciones y comentarios al haber visto los datos y análisis presentados por los ponentes. El sentimiento es un producto mezclado: por una parte, intelectual y, por otra, afectivo.

Sin duda, los esfuerzos y las investigaciones representados por los artículos de estos dos volúmenes demuestran que la arqueología histórica se ha establecido en el Perú. A la vez, afirmamos que la cultura y los procesos sociohistóricos del Perú virreinal tienen bastante que ofrecer a la subdisciplina 
de la arqueología histórica. Concluimos notando la referencia planteada hace unos cinco años en la introducción de un volumen especial de la revista Historical Archaeology, dedicado al tema de la arqueología histórica en Sudamérica. Los editores, Juan G. Martín, Alasdair Brooks y Tania Andrade Lim, escribieron sobre el SIAH: «Recientemente, se han visto indicadores claros de un crecimiento de la arqueología histórica en los Andes centrales. El inaugural simposio internacional de la arqueología histórica peruanista, Posibilidades y Perspectivas para una Arqueología Histórica en el Perú, [...] ocurrió en Lima en el 2010 [...]. Los objetivos del simposio incluyeron facilitar en definir la arqueología histórica andina y establecer trayectorias de investigación para el desarrollo del futuro de la disciplina a lo largo de los Andes centrales. Esperamos que la publicación de las ponencias presentadas en este simposio importante salga próximamente» (Martín et al. 2012: 5-6, traducción propia) ${ }^{10}$. Nosotros esperamos que el presente conteste esta llamada de nuestros colegas. También, anticipamos el creciente número de conversaciones que florecerán entre los investigadores aquí representados y los de las investigaciones de la arqueología histórica actuales y futuras. Desde nuestro punto de vista, el panorama es amplio y alentador.

\section{Agradecimientos}

La publicación de estos dos volúmenes no habría sido posible sin el apoyo de un gran número de colegas e instituciones. Ante todo, queremos agradecer a los doctores Luis Jaime Castillo (director del Boletín de Arqueología PUCP), Ana Cecilia Mauricio y Francesca Fernandini por su apoyo constante en la edición de estos dos volúmenes. La edición de esta publicación ha sido apoyada parcialmente por Brown University, Brigham Young University y Queen's University Belfast. El Simposio Internacional de Arqueología Histórica fue realizado con el apoyo generoso de la Universidad Ricardo Palma y la actual directora del Centro Cultural Ccori Wasi, doctora Sandra Negro, además del David Rockefeller Center of Latin American Studies (Harvard University). Agradecemos a varios autores y comentaristas en el simposio, cuyos trabajos no se encuentran representados entre esta colección: Marco Curatola, Thomas Cummins, Juan Carlos Estenssoro, Elmo León, Krzysztof Makowski, Sandra Negro, Juan Ossio, Karen Spalding, Frank Salomon, Jacob Sauer, Daniel Schavelzon, Edward Swenson y Rafael Vega-Centeno.

\section{Notas}

${ }^{1}$ En este volumen y el siguiente, incluimos un total de cuatro artículos que tratan investigaciones realizadas fuera del Perú actual, pero dentro del virreinato del Perú: Urbina y Uribe (Tarapacá, Chile), Weaver, Van Buren (Porco, Bolivia) y Jamieson (Riobamba, Ecuador).

${ }^{2}$ Por ejemplo, sugerimos que es imposible entender por completo la formación de redes de intercambio en el sur del Perú durante los siglos XVI y XVII (un tema abordado por Prudence Rice en su aporte a esta colección) sin considerar los sitios de Potosí y Porco, actualmente localizados en Bolivia y discutidos en esta colección por Mary Van Buren y Brendan Weaver. Por supuesto, lo inverso también es cierto: las economías de Porco y Potosí difícilmente pueden entenderse sin referencias a la extracción de mercurio en Huancavelica; la producción de vino y otros productos en valles peruanos como Moquegua; la migración temporal de los mitayuq desde territorios actualmente encontrados dentro de los bordes de Perú y Chile; ni hablar del comercio de esclavos que unía a Potosí con Lima, Cartagena, Portobelo y el mundo atlántico (como también demuestra el artículo de Alexander Menaker en el siguiente volumen). Sin duda, hay líneas importantes de investigación en los estudios de la época colonial y la época republicana, cuyos enfoques son más regionales, pero quisiéramos subrayar la importancia notable de la escala internacional a la arqueología de los períodos colonial y republicano. 
${ }^{3}$ Funari (1999) sugiere que el uso de la etiqueta "histórica» para referir a la arqueología post siglo $\mathrm{XV}$ es un turno de frase particularmente norteamericana, que refleja la distancia presunta entre la arqueología y la historia en las academias estadounidense y canadiense. Según su evaluación, Latinoamérica es la única otra región del mundo que comparte esta visión, pero cabe notar que también se encuentra en trabajos producidos en otros Estados-naciones fundados por colonos anglófonos, particularmente, Australia y Nueva Zelanda (v.g. Connah 1998; Murray y Allen 1986).

${ }^{4}$ Algunos comentaristas han sugerido que la arqueología en el Perú fue fuertemente influenciada durante estos ańos por el discurso cultural del gobierno de Juan Velasco Alvarado (1968-1975), que exaltó al Imperio inka como una fuente de identidad y orgullo nacional (Oyuela Caycedo et al. 1997; Patterson 1994). No obstante, si bien la arqueología social de Luis Lumbreras (1974) y otros no dedicó mucha atención a los períodos poscoloniales, tampoco expresó ser explícitamente contraria a la idea. Si bien el gobierno de Velasco no proporcionó apoyo material a la arqueología de los sitios históricos en el Perú, tampoco suprimió los intereses de los estudiosos peruanos en el tema: muchos de los trabajos y publicaciones pioneros del Seminario de Arqueología de la Pontificia Universidad Católica del Perú fueron realizados durante los ańos del gobierno de Velasco.

5 «La arqueología histórica tiene varias definiciones. Una de las más lamentables — que, ciertamente, no es la verdad [...] — es que la arqueología histórica es la forma más costosa que hay de aprender algo que ya supimos» («Historical archaeology has several definitions. One of the more unfortunate ones-which is certainly not true ...- is that historical archaeology is the most expensive way in the world to learn something we already know», Deetz 1991: 1; traducción propia). Esta caracterización de la arqueología histórica aparentemente tiene sus orígenes en los comentarios de un historiador. Véase Wass (2013) en http://www.polyolbion.org.uk/Leicester/MA.html.

${ }^{6}$ No obstante, las investigaciones arqueológicas de sitios históricos han desmentido esta concepción desde hace mucho tiempo. De hecho, la historia entera de los Inka y Tawantinsuyu se encuentra actualmente en un estado de revisión por causa del macizo de datos arqueológicos acumulados durante las últimas décadas (v.g. Pärssinen y Siiriäinen 1997; Cornejo 2014; Covey 2015; D’Altroy et al. 2007; Tantaleán 2015; Marsh et al. 2017).

7 Juan Ossio también hizo un comentario muy similar durante el evento de SIAH.

${ }^{8}$ Con ello, se alude a la "presentación formal», «prácticas sociales reiterativas» (véase Austin 1962; Inomata y Coben [eds.] 2006).

${ }^{9}$ https://sha.org/about-us/

${ }^{10}$ «More recently, there have been clear signs of a growth in historical archaeology in the central Andes. The first-ever international symposium on Peruvian Historical Archaeology, Posibilidades y Perspectivas para una Arqueología Histórica en el Perú [...] was held in Lima in 2010 [...]. The goals of this conference included helping to define Andean historical archaeology and to set research agendas for the discipline's future development across the central Andes [...]. It is to be hoped that publication of the papers presented at this important symposium will be forthcoming» (Martín et al. 2012: 5-6). 


\section{REFERENCIAS}

Abercrombie, T.

1998 Pathways of memory and power: Ethnography and history among an Andean people, University of Wisconsin Press, Madison.

Acevedo Basurto, $S$.

1986 Trayectoria de la cerámica vidriada en el Perú, en: F. Stastny y S. Acevedo (eds.), Vidriados y mayólica del Perú, 19-31, Museo de Arte y de Historia/Universidad Nacional Mayor de San Marcos, Lima.

Aguirre, C.

2005 Breve historia de la esclavitud en el Perú: Una herida que no deja de sangrar, Fondo Editorial del Congreso del Perú, Lima.

Andrews, G. R.

2016 Afro-Latin America: Black lives, 1600-2000, Harvard University Press, Cambridge.

Arellano, C.

1999 Quipu y tocapu: Sistemas de comunicación Incas. en: F. Pease (ed.), Los Incas: Arte y simbolos, 215-261, Banco de Crédito del Perú, Lima.

Arrelucea Barrantes, M.

2009 Replanteando la esclavitud: Estudios de etnicidad y género en Lima borbónica, Cadet, Lima.

Arrieta Álvarez, A., C. Arellano Hoffmann, A. Castañeda y J. Polo

1975 Cerámica de transición: Huaca Palomino, valle del Rímac, Boletín del Seminario de Arqueología (15-16), 159-166.

Atalay, S., Clauss, L. R., McGuire, R. H. y J. R. Welch (eds.)

2014 Transforming archaeology: Activist practices and prospects, Left Coast Press, New York. https://doi.org/ $10.4324 / 9781315416533$

Austin, J. L.

1975 How to do things with words, Oxford University Press, Oxford.

Bárcena, J. R.

1993 Las investigaciones arqueológicas e históricas y las posibilidades económicas del Rescate y valorización del patrimonio cultural, Boletín de La Bolsa de Comercio de Mendoza 351, 3-4.

Bauer, B. S. y A. Coello

2007 The hospital of San Andrés (Lima, Peru) and the search for the royal mummies of the Incas, Fieldiana Anthropology 39, 1-31. https://doi.org/10.3158/0071-4739(2007)188[1:thosal]2.0.co;2

Beck, C. M., E. E. Deeds, S. Pozorski y T. Pozorski

1983 Pajatambo: An 18th century roadside structure in Peru, Historical Archaeology 17 (1), 54-68.

Berdichewsky Scher, B. y M. Calvo de Guzmán

1972 Excavaciones en cementerios indigenas de la región de Calafquén, VI Congreso de Arqueología Chilena, 529-558, Arica.

Bowser, F. P.

1974 The African slave in colonial Peru 1524-1650, Stanford University Press, Stanford.

Brockington, L. G.

2006 Blacks, Indians, and Spaniards in the eastern Andes: Reclaiming the forgotten in colonial Mizque, 1550-1782, University of Nebraska Press, Lincoln.

Brokaw, G.

2010 A history of the khipu, Cambridge University Press, Cambridge.

Cárdenas, $\mathrm{M}$.

1970 Ocupación española de una huaca del valle de Lima: casa en la plataforma superior de la Huaca Tres Palos, Boletín del Seminario de Arqueología 5, 40-49. 
1971 Huaca Palomino, valle de Rímac: Fragmentería vidriada fina con decoración en colores, Boletín del Seminario de Arqueología 10, 61-67.

1973 Cerámica de transición: Huaca Palomino, valle del Rímac, Boletín del Seminario de Arqueología 14, 30-34.

Chase, Z. J.

2015 What is a wak'a? When is a wak'a?, en: T. L. Bray (ed.), The archaeology of wak'as: explorations of the sacred in the pre-Columbian Andes, 75-126, University Press of Colorado, Boulder. https://doi. org/10.5876/9781607323181.c004

2016 Performing the past in the historical, ritual, and mythological landscapes of Huarochirí, Peru (ca. AD 1400-1700), tesis de doctorado, Departamento de Antropología, Universidad de Chicago, Chicago.

2017 Past-forward: Archaeology and history of late prehispanic and early colonial Andean past making, en: E. Swenson y A. Roddick (eds.), Constructions of time and history in the pre-Columbian Andes, University Press of Colorado, Boulder.

Christian, D.

2011 Maps of time: An introduction to big history, University of California Press, Berkeley.

Chuhue, R., L. Ning Na y A. Coello (eds.)

2012 La inmigración China al Perú: Arqueología, historia y sociedad, Universidad Ricardo Palma Instituto Confucio, Editorial Universitaria, Lima.

Cogorno, G.

1970 Cerámica de transición: Huaca Palomino, valle del Rímac, Boletín del Seminario de Arqueología 5, 1-39.

Connah, G.

1998 Pattern and purpose in historical archaeology, Australasian Historical Archaeology 16, 3-7.

Cornejo, L.

2014 Sobre la cronología de la imposición cuzqueña en Chile, Estudios Atacameños 47, 101-116. https://doi. org/10.4067/s0718-10432014000100007

Covey, R. A.

2015 Inka imperial intentions and archaeological realities in the Peruvian highlands, en: I. Shimada (ed.), The Inka empire: A multidisciplinary approach, 83-95, University of Texas Press, Austin.

Cummins, T. B. F.

1994 Representation in the 16th century and the colonial image of the Inca, en: E. H. Boone, y W. Mignolo (eds.), Writing without words: Alternative literacies in Mesoamerica and the Andes, 188-219, Duke University Press, Durham. https://doi.org/10.1215/9780822379263-009

2002 Forms of Andean colonial towns, free will, and marriage, en: C. L. Lyons y J. K. Papadopoulos (eds.), The archaeology of colonialism, 199-240, Getty Research Institute, Los Angeles.

Crespo, A. R.

1977 Esclavos negros en Bolivia, Academia Nacional de Ciencias de Bolivia, La Paz.

Curatola, M. y J. C. de la Puente (eds.)

2013 El quipu colonial: Estudios y materiales, Pontificia Universidad Católica del Perú, Lima.

Cushner, N. P.

1975 Slave mortality and reproduction on Jesuit haciendas in colonial Peru, The Hispanic American Historical Review 55 (2), 177-199. https://doi.org/10.2307/2512093

D'Altroy, T. N., V. I. Williams y A. M. Lorandi

2007 The Inkas in the southlands, en: R. L. Burger, C. Morris y R. Matos Mendieta (eds.), Variations in the expression of Inka power, 85-133, Dumbarton Oaks, Washington, D.C.

Dawdy, S. L.

2016 Profane archaeology and the existential dialectics of the City, Journal of Social Archaeology 16(1), 32-55. https://doi.org/10.1177/1469605315615054

Deetz, J.

1991 Introduction: Archaeological evidence of sixteenth- and seventeenth-century encounters, en: L. Falk (ed.), Historical archaeology in global perspective, 1-9, Smithsonian Institution Press, Washington D.C. 
deFrance, S. D.

1996 Iberian foodways in the Moquegua and Torata Valleys of southern Peru, Historical Archaeology 30 (3), 20.48 .

DeFrancis, J.

1989 Visible speech: The diverse oneness of writing systems, University of Hawaii Press, Honolulu.

De León, J. P.

2015. The land of open graves: Living and dying on the migrant trail, University of California Press, Berkeley.

Donnan, C. B.

2011 Chotuna and Chornancap: Excavating an ancient Peruvian legend, Cotsen Institute of Archaeology Press, Los Angeles.

Druc, I.

2013. What Is Local? Looking at ceramic production in the Peruvian highlands and beyond, Journal of Anthropological Research 69, 485-513. https://doi.org/10.3998/jar.0521004.0069.404

Ferguson, L. G.

1992 Uncommon Ground: Archaeology and Colonial African-America, Smithsonian Institution Press, Washington, DC.

Funari, P. P. A.

1997 Archaeology, history, and historical archaeology in South America, International Journal of Historical Archaeology 1 (3), 189-206.

1999 Historical archaeology from a world perspective, en: P. P. A. Funari, M. Hall y S. Jones (eds.), Historical archaeology: Back from the Edge, 37-66, Routledge, Londres.

2003 Conflict and the Interpretation of Palmares, a Brazilian Runaway Polity, Historical Archaeology 37 (3), 81-92. https://doi.org/10.1007/bf03376613

2006 Conquistadors, plantations, and quilombo: Latin America in historical archaeological context, en: M. Hall y S. W. Silliman (eds.), Historical Archaeology, 209-229, Blackwell, Malden.

Funari, P. P. A. y C. E. Orser, Jr. (eds.)

2015 Current perspectives on the archaeology of African slavery in Latin America, Springer, New York.

Funari, P. P. A. y A. Zarankin

2006 Arqueología de la represión y la resistencia en América Latina (1960-1980), Editorial Brujas, Buenos Aires.

Fusco Zambetogliris, N.

1996 Crónica de un impacto anunciado, Historical Archaeology in Latin America 16, 1-10.

Giersz, M. y C. Pardo (eds.)

2014 Castillo de Huarmey: El mausoleo imperial Wari, Asociación Museo de Arte de Lima, Lima.

Goody, J.

1986 The logic of writing and the organization of society, Cambridge University Press, Cambridge. https://doi. org/10.1017/cbo9780511621598

2000 The power of written tradition, Smithsonian Institution Press, Washington, D.C.

Guimarães, C. M.

1992 Esclavage, quilombos et archéologie, Les Dossiers d'Archeologie 169, 67.

Guimarães, C. M. y A. L. Duarte Lanna

1980 Arqueologia de quilombos em Minas Gerais, Pesquisas, Antropologia 31, 147-164.

Harth-Terré, E.

1973 Negros e indios: Un estamento social ignorado del Perú colonial, Liberia/Editorial Juan Mejía Baca, Lima.

Hyland, S. P.

2002 Woven words: The royal khipu of Blas Valera, en: J. Quilter y G. Urton (eds.), Narrative threads: Accounting and recounting in Andean khipu, 151-170, University of Texas Press, Austin.

2014 Ply, markedness, and redundancy: New evidence for how Andean khipus encoded information, American Anthropologist 116, 643-648. https://doi.org/10.1111/aman.12120

Inomata, T. y L. A. Coben (eds.)

2006 Archaeology of performance: Theaters of power, community, and politics, AltaMira Press, Lanham. 
Jamieson, R. W.

2005 Colonialism, social archaeology and lo andino: Historical archaeology in the Andes, World Archaeology 37 (3), 352-372. https://doi.org/10.1080/00438240500168384

Jouve Martín, J. R.

2008 Esclavos de la ciudad letrada: Esclavitud, escritura y colonialismo en Lima (1650-1700), Instituto de Estudios Peruanos, Lima.

Kern, A. A.

1991 Sociedade barroca e Missões Guaranis: Do confronto À complementaridade, Actas Do Primeiro Congresso Internacional Do Barroco, 445-465.

1994 Antecedentes indigenas, Editora da Universidade, Universidade Federal do Rio Grande do Sul, Porto Alegre.

Lane, $\mathrm{K}$.

2002 Quito 1599: City and colony in transition, University of New Mexico Press, Albuquerque.

Lecoq, P.

2013 Can the Inca site of Choqek' iraw be considered and agro-pastoral calendar? Nawpa Pacha 33 (1), 43-69. https://doi.org/10.1179/0077629713z.0000000003

Leone, M. P., P. B. Potter y P. A. Shakel

1987 Toward a critical archaeology, Current Anthropology 28, 283-302.

Lumbreras, L.

1974 La arqueología como ciencia social. Lima: Ediciones Histar.

\section{Lightfoot, $\mathrm{K}$.}

1995 Culture contact studies: Redefining the relationship between prehistoric and historical, American Antiquity 60(2), 199-217. https://doi.org/10.2307/282137

Little, B. J. y Shackel, P. A. (eds.)

2007 Archaeology as a tool of civic engagement, Altamira, Lanham.

\section{Makowski, K.}

2009 Viru-Moche relations: Technological identity, stylistic preferences, and the ethnic identity of ceramic manufacturers and users, en: J.-F. Millaire y M. Morlion (eds.) Gallinazo: An early cultural tradition on the Peruvian North Coast, 33-60, Cotsen Institute of Archaeology Press, Los Angeles.

Marsh, E. J., R. Kidd, D. Ogburn y V. Durán

2017 Dating the expansion of the Inca Empire: Bayesian models from Ecuador and Argentina, Radiocarbon 2017, 1-24. https://doi.org/10.1017/rdc.2016.118

Martín, J. G., A. Brooks y A. Andrade Lima

2012 Crossing borders and maintaining identities: Perspectives on current research in South American historical archaeology, Historical Archaeology 46 (3), 1-15. https://doi.org/10.1007/bf03376867

McGuire, R. H.

1992 A Marxist archaeology, Academic Press, New York.

2008 Archaeology as political action, University of California Press, Berkeley.

McKinley, M.

2010 Fractional freedoms: Slavery, legal activism \& ecclesiastical courts in colonial Lima, 1593-1700, Law and History Review 28 (3), 749-790. https://doi.org/10.1017/s0738248010000623

2012 Till death do us part: Testamentary manumission in seventeenth-century Lima, Slavery and abolition: $A$ Journal of Slave and Post Slave Studies 33 (3), 381-401. https://doi.org/10.1080/0144039x.2012.668302

2014 Standing on shaky ground: Criminal jurisdiction and ecclesiastical immunity in seventeenth-century Lima, University of California-Irvine Law Review 5 (3), 101-34.

Miasta Gutiérrez, J.

1985 Arqueología histórica en Huarochirí: Santo Domingo de Los Olleros, San José de Los Chorrillos y San Lorenzo de Quinti, vols. I y II, Universidad Nacional Mayor de San Marcos, Seminario de Historia Rural Andina, Lima.

2006 Arqueología histórica en Huarochiri: Santo Domingo de Los Olleros, San José de Los Chorrillos y San Lorenzo de Quinti, Consejo Nacional de Ciencia, Tecnología e Innovación Tecnológica, Lima. 
Millones, L.

1971 Gente negra en el Perú: Esclavos y conquistadores, América Indígena 31 (3), 593-624.

1973 Minorías étnicas en el Perú, Pontifica Universidad Católica del Perú, Lima.

Mogrovejo, J. D.

1996 Arqueología urbana de evidencias coloniales en la ciudad de Lima, Cuadernos de Investigación, Pontificia Universidad Católica del Perú/Instituto Riva-Agüero, Lima.

Murray, T. y J. Allen

1986 Theory and the Development of Historical Archaeology in Australia, Archaeology in Oceania 21 (1), 85-93. https://doi.org/10.1002/j.1834-4453.1986.tb00128.x

Mytum, H.

2016 A short history of the society for post-Medieval archaeology, Post-Medieval Archaeology 50 (1), 6-18. https://doi.org/10.1080/00794236.2016.1160626

Orser, C. E. y P. P. A. Funari

2001 Archaeology and slave resistance and rebellion, World Archaeology 33 (1), 61-72. https://doi.org/ $10.1080 / 00438240126646$

O'Toole, R. S.

2012 Bound lives: Africans, Indians, and the making of race in colonial Peru, University of Pittsburgh Press, Pittsburgh.

Oyuela-Caycedo, A., Anaya, C. Elera y L. M. Valdez

1997 Social Archaeology in Latin America?: Comment to T. C. Patterson, American Antiquity 62 (2), $365-374$.

Pärssinen, M. y A. Siiriäinen

1997 Inka-style ceramics and their chronological relationship to the Inka expansion in the southern Lake Titicaca Area (Bolivia), Latin American Antiquity 8, 255-271. https://doi.org/10.2307/971655

Patterson, T. C.

1994 Social archaeology in Latin America: An appreciation, American Antiquity 59 (3), 531-537.

Pedrotta, V. y F. Gómez

1998 Historical archaeology: An outlook from the Argentinean Pampas, International Journal of Historical Archaeology 2 (2), 113-131.

Pino, J. L.

2014 El tocapu que narra el viaje del Sol en el mes de agosto: La arquitectura inca como representación calendárica del orden. Una vision de Huánuco Pampa, en: C. Arellano Hoffmann (ed.), Sistemas de notación inca: Quipu y tocapu, 359-393, Ministerio de Cultura, Lima.

Politis, G. G.

2003 The theoretical landscape and the methodological development of archaeology in Latin America, American Antiquity 68 (2), 245-272. https://doi.org/10.2307/3557590

Poujade, R. A.

1992 Poblamiento prehistórico y colonial de Misiones, Estudios Ibero-Americanos 18, 29-69.

Quilter, J. y G. Urton (eds.)

2002. Narrative threads: Accounting and recounting in Andean Khipu, University of Texas Press, Austin.

Quilter, J., M. Zender, K. Spalding, R. Franco, C. Gálvez y J. Castañeda

2010 Traces of a lost language and number system discovered on the North Coast of Peru, American Anthropologist 112 (3), 357-369.

Raimondi, A.

1874 El Perú. Parte preliminar, tomo I, Imprenta del Estado, Lima.

Rappaport, J.

1994 Object and alphabet: Andean Indians and documents in the colonial Period, en: E.H. Boone y W. Mignolo (eds.), Writing without words: Alternative literacies in Mesoamerica and the Andes, 271-291, Duke University Press, Durham. 


\section{Rappaport, J. y T. Cummins}

1994 Literacy and power in colonial Latin America, en: C. C. Bond y A. Gilliam (eds.), Social construction of the past: Representation as Power, 89-112. Routledge, New York.

2011 Beyond the lettered city: Indigenous literacies in the Andes, Duke University Press, Durham. https://doi. org/10.1215/9780822394754

Rice, P. M.

1994 The kilns of Moquegua, Peru: Technology, excavations, and functions, Journal of Field Archaeology 21 (3), 325-344. https://doi.org/10.2307/530334

1996 The archaeology of wine: the wine and brandy haciendas of Moquegua, Peru, Journal of Field Archaeology 23 (2), 187-204. https://doi.org/10.2307/530503

1997a Wine and brandy production in colonial Peru: A historical and archaeological investigation, The Journal of Interdisciplinary History 27 (3), 455-479. https://doi.org/10.2307/205915

$1997 \mathrm{~b}$ Tin-enameled wares of Moquegua, Peru, en: J. Gasco, G. C. Smith y P. Fournier García (eds.), Approaches to the historical archaeology of Mexico, Central and South America, 173-180, Institute of Archaeology, UCLA, Los Angeles.

2012a Torata Alta: An Inka administrative center and Spanish colonial reducción in Moquegua, Peru, Latin American Antiquity 23 (1), 3-28. https://doi.org/10.7183/1045-6635.23.1.3

2012b Vintage Moquegua: History, wine, and archaeology on a colonial Peruvian periphery, University of Texas Press, Austin.

2013 Political-ecology perspectives on New World loza (Majolica), International Journal of Historical Archaeology 17 (4), 651-683. https://doi.org/10.1007/s10761-013-0238-x

Rice, P. M. y S. L. Van Beck

1993 The Spanish colonial kiln tradition of Moquegua, Peru, Historical Archaeology 27 (4), 65-81. https://doi. org/10.1007/bf03373574

Rowe, J. H.

1998 Max Uhle y la idea del tiempo en la arqueología americana, en: P. Kaulicke (ed.), Max Uble y el Perú antiguo, 5-24, Pontificia Universidad Católica el Perú, Lima.

Salomon, F.

2002 Un-ethnic ethnohistory: On Peruvian peasant historiography and the ideas of autochthony, Ethnohistory 49 (3), 475-506. https://doi.org/10.1215/00141801-49-3-475

2006 Los quipucamayos: El arte del khipu en una comunidad campesina moderna, Instituto Francés de Estudios Andinos, Lima.

Salomon, F. y M. Nińo-Murcia

2011 The lettered mountain: A Peruvian village's way with writing, Duke University Press, Durham. https://doi. org/10.1215/9780822394341

Salomon, F. y G. L. Urioste

1991 The Huarochiri manuscript: A testament of ancient and colonial Andean religion, University of Texas Press, Austin.

Sanoja Obediente, M.

1978 Proyecto Orinoco: Excavación en el sitio arqueológico de Los Castillos de Guayana, territorio federal Delta Amacuro, Venezuela, Actas del Séptimo Congreso Internacional para el Estudio de las Culturas Precolombinas de Las Antillas Menores, 157-168, Universidad Central/Centre de Recherches Caraibes, Université de Montreal, Caracas/Montreal.

Schávelzon, D.

1988 Tipología de la loza arqueológica de Buenos Aires (1780-1900), Programa de Arqueología Urbana, Buenos Aires.

1991 Arqueología histórica de Buenos Aires: La cultura material porteña de los siglos XVIII y XIX, Corregidor, Buenos Aires.

1998 Las ruinas de San Francisco: Arqueología e historia, Municipalidad de la Ciudad de Mendoza/Editorial Tintar, Mendoza.

2000 The historical archaeology of Buenos Aires: A city at the end of the world, Kluwer Academic / Plenum, New York.

Scott, E. M. (ed.)

1994 Those of little note: Gender, race, and class in historical archaeology, University of Arizona Press, Tucson. 
Silverman, H. y W. H. Isbell (eds.)

2008 Handbook of South American archaeology, Springer, New York. https://doi.org/10.1007/978-0-38774907-5

\section{Singleton, T. A.}

1995 The archaeology of slavery in North America, Annual Review of Anthropology 24, 119-140. https://doi. org/10.1146/annurev.anthro.24.1.119

\section{Singleton, T. A. y M. A. Torres de Souza}

2010 Archaeologies of the African diaspora: Brazil, Cuba, and the United States, en: T. Majewski y D. Gaimster (eds.), International handbook of historical archaeology, 449-469, Springer, New York. https:// doi.org/10.1007/978-0-387-72071-5_26

Smail, D. L.

2005 In the grip of sacred history, The American Historical Review 110 (5), 1337-1361. https://doi.org/10.1086/ ahr.110.5.1337

2007 On deep history and the brain, University of California Press, Berkeley.

Smith, G.

1991 Heard it through the grapevine: Andean and European contributions to Spanish colonial culture and viticulture in Moquegua, Peru, tesis de doctorado, Departamento de Antropología, University of Florida, Gainesville.

1997 Hispanic, Andean, and African influences in the Moquegua Valley of Southern Peru, Historical Archaeology 31 (1), 74-83. https://doi.org/10.1007/bf03377257

Stastny, F.

1981 Las artes populares del Perú, Ediciones Edubanco, D.L., Madrid.

Stastny, F. y S. Acevedo

1986 Vidriados y mayólica del Perú, Universidad Nacional Mayor de San Marcos, Facultad de Letras y Ciencias Humanas/Museo de Arte y de Historia, Lima.

Swenson, E. y A. Roddick (eds.)

2017 Constructions of time and history in the pre-Columbian Andes, University Press of Colorado, Boulder.

Tantaleán, $\mathrm{H}$.

2015 El imperio inca: Indicadores arqueológicos de un estado expansivo andino, Inka Llaqta 4, 9-42.

2016 Una Historia de la arqueología peruana, Instituto de Estudios Peruanos, Lima.

von Tschudi, J. y M. E. de Rivero

1851 Antigüedades peruanas, Imprenta Imperial del Corte y del Estado, Viena.

Thomas, J.

2004 Archaeology and modernity, Routledge, Londres. https://doi.org/10.4324/9780203491119

Trouillot, M. R.

1995 Silencing the past: Power and the production of history, Beacon Press, Boston.

2003 Global transformations: Anthropology and the modern world, Palgrave Macmillan, New York.

Urton, G.

2008 The Inca khipu: Knotted-cord record keeping in the Andes, en: H. Silverman y W. H. Isbell (ed.), Handbook of South American archaeology, 831-844, Springer, New York. https://doi.org/10.1007/978-0387-74907-5_41

2017 Writing the history of an ancient civilization without writing: Reading the Inka khipus as primary sources, Journal of Anthropological Research 73(1), 1-21. https://doi.org/10.1086/690611

Urton, G. y C. Brezine

2005 Khipu accounting in ancient Peru, Science 309, 1065-1067. https://doi.org/10.1126/science.1113426

Urton, G. y A. Chu

2015 The Inkawasi khipu archive: An inka state storage facility and accounting center on the south coast of Peru, Latin American Antiquity 26 (4), 512-29. 


\section{Van Buren, M.}

1993 Community and empire in southern Peru: The site of Torata Alta under Spanish rule, tesis de doctorado, Departamento de Antropología, University of Arizona, Tucson.

1996 Rethinking the vertical archipelago: Ethnicity, exchange, and history in the south-central Andes, American Anthropologist 98, 338-351. https://doi.org/10.1525/aa.1996.98.2.02a00100

2010 The archaeological study of Spanish colonialism in the Americas, Journal of Archaeological Research 18 (2), 151-201. https://doi.org/10.1007/s10814-009-9036-8

Villacorta, L. F.

2012 Antonio Raimondi, archaeology, and national discourse: Representations and meanings of the past in Nineteenth-century Peru, en: J. Pillsbury (ed.), Past presented: Archaeological illustration and the Ancient Americas, 173-206, Dumbarton Oaks, Washington, D.C.

Wass, $S$.

2013 Material prepared as part of Leicester University's M.A, Programme in Historical Archaeology. http:// www.polyolbion.org.uk/Leicester/MA.html, accedido: 5 Marzo 2017.

Weaver, B. J. M.

2015 Fruit of the vine, work of human hands»: An archaeology and ethnohistory of slavery on the on the Jesuit wine haciendas of Nasca, Peru, tesis de doctorado, Departamento de Antropología, Vanderbilt University, Nashville.

2016 Perspectivas para el desarrollo de una arqueología de la diáspora africana en el Perú: Resultados preliminares del Proyecto Arqueológico Haciendas de Nasca, Allpanchis 80 ( $2^{\circ}$ semestre, 2012), 85-120.

Zarankin, A.

1995 Arqueologia histórica urbana en Santa Fe La Vieja: El final del principio (edición de A. Stanley), Arqueología Histórica en América Latina 10, University of South Carolina Press, Columbia.

Zarankin, A. y M. A. Salerno

2008 «Looking south»: Historical archaeology in South America, Historical Archaeology 42 (4), 38-58. https:// doi.org/10.1007/bf03377153

Zimmerman, L. J., C. Singleton y J. Welch

2010 Activism and creating a translational archaeology of homelessness, World Archaeology 42, 443-454. https://doi.org/10.1080/00438243.2010.497400 